\title{
Carnets
}

Revue électronique d'études françaises de l'APEF

Deuxième série - 5 | 2015

Imaginaires de guerre et autres conflits

\section{Guerres du futur, conflits du présent}

Irène Langlet

\section{(2) OpenEdition}

\section{Journals}

Édition électronique

URL : http://journals.openedition.org/carnets/311

DOI : 10.4000/carnets.311

ISSN : 1646-7698

Éditeur

APEF

\section{Référence électronique}

Irène Langlet, "Guerres du futur, conflits du présent », Carnets [En ligne], Deuxième série - 5 | 2015, mis en ligne le 30 novembre 2015, consulté le 30 avril 2019. URL : http://journals.openedition.org/ carnets/311; DOI : 10.4000/carnets.311

Ce document a été généré automatiquement le 30 avril 2019.

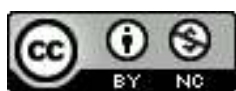

Carnets est mis à disposition selon les termes de la licence Creative Commons - Atribution - Pas d'utilisation commerciale 4.0 International. 


\title{
Guerres du futur, conflits du présent
}

\author{
Irène Langlet
}

1 En 1987, Denis Guiot écrivait : «La science-fiction n'en finit pas de parler de guerre » (Guiot, 1987). Jacques Goimard, lui, dans une préface signée deux ans plus tôt pour le volume « Histoire de guerres futures » de la Grande anthologie de la science-fiction, estimait que : « La guerre est un thème universel, dont la science-fiction ne saurait revendiquer le monopole.» Pour Goimard, il s'agissait de relativiser l'importance d'un thème responsable, à ses yeux, du mépris dans lequel on pouvait tenir la science-fiction encore en 1985 : «La science-fiction ne mérite sans doute pas toujours les réactions phobiques qu'elle continue de susciter chez ses fidèles ennemis. Pourtant le fait est qu'elle parle souvent de la guerre, si souvent qu'il y a de quoi s'interroger. » (Goimard, 1985 : 5). Il n'est pas nécessaire de prendre position dans ce débat pour observer que la guerre est si nécessaire à la science-fiction qu'elle est l'invariant thématique d'au moins deux de ses sous-genres les plus importants: le space opera et le récit post-nucléaire. Une fois cela dit, on n'aura pas pour autant défini un genre populaire militariste, bien au contraire; la seule existence d'une étiquette de "science-fiction militariste » parmi les multiples sousdivisions du genre dit à elle seule que la science-fiction n'accomplit pas que des récits de guerres, a fortiori dans l'espace français.

2 Ces deux sous-genres sont toutefois fort utiles pour comprendre deux époques de l'histoire littéraire, qui sont aussi deux époques de l'histoire culturelle de la guerre en Occident. Ainsi, le space opera narre des aventures dans l'espace; il articule le récit d'un voyage extraordinaire avec des scénarios de combats dans l'espace, soit contre des races extra-terrestres, soit entre humains. Ses invariants structurels sont la vastitude des théâtres des combats, la technologie fantastique mise en oeuvre, les ramifications infinies des intrigues diplomatiques présidant aux conflits, et les organisations politiques plus ou moins originales chapeautant les sociétés mises en jeu (en fait, la plupart du temps des «Fédérations » ou des « Empires »). Le post-nucléaire, en revanche, se passe sur Terre et raconte la vie après la catastrophe qui a, dans la plupart des scénarios, remis à zéro les techniques et les sociétés en même temps qu'elle altérait durablement les milieux naturels. Un survivalisme anime les scénarios, ainsi qu'une violence sociale et naturelle qui fait écho à celle qui est à l'origine du monde fictif. 
3 Dans un cas, il s'agit donc de s'immerger complaisamment dans le récit de guerre, et d'exporter au-delà de la Terre son exaltation lyrique et son idéologie héroïque, à l'aide d'une technologie triomphante mise au service de l'épopée militaire. Dans l'autre, il s'agit au contraire de se voir confiné à une Terre dégradée, où la guerre minimalisée répète et diffracte en violence quotidienne le souvenir d'une sorte de guerre absolue, réduite à un geste de destruction totale (le motif $\mathrm{du}$ «bouton rouge » qu'il suffit de pousser pour déclencher «la bombe »). Du space opera au post-nucléaire, vingt ans d'écart: des années 1930 aux années 1950, et une guerre dite, pour la première fois de l'Histoire, non seulement «mondiale » mais «totale»; deux ordres du monde sensiblement différents, passant de la décomposition des Empires européens après la $1^{\text {re }}$ Guerre Mondiale à l'instauration de la Guerre Froide après 1945. Mais c'est sur le plan des contextes culturels que le contraste concerne le plus notre lecture. On restera attentif en effet, dans ce panorama thématique, à ce que Letourneux répète avec insistance dans un récent article : "le récit de guerre future n'existe que dans le dialogue avec les autres séries culturelles " (Letourneux, 2015: 225). La série culturelle qui voit s'épanouir le space opera, c'est la fiction populaire bon marché alimentant l'industrie culturelle américaine des pulp magazines (revue Amazing Stories par exemple) et des premiers comics. Celle du postnucléaire, c'est plutôt la généralisation du cinéma de série $B$, la maturation aux États-Unis d'une seconde génération d'auteurs et de revues (Astounding Science Fiction, puis Galaxy et The Magazine of Science Fiction and Fantasy), des scénarios à la fois plus plausibles et plus ironiques, voire critiques. Le space opera ne disparait pourtant pas ; l'écriture de la guerre science-fictionnelle est donc, dans le biotope américain lui-même, le lieu d'une tension culturelle. C'est à partir de cette tension que la «science-fiction " s'implante en France près 1945 , et s'hybride avec les problématiques locales.

4 L'étude de Letourneux se consacre à une littérature d'avant 1914 ; nous observerons ici celle $\mathrm{du}$ second $\mathrm{xx}^{\mathrm{e}}$ siècle. L'Entre-Deux Guerres n'est pourtant certes pas une période sans intérêt ; retenons, entre autres, Théo Varlet (L'Agonie de la Terre, 1922), Régis Messac ( Quinzinzinzili, 1935), Jacques Spitz (La Guerre des Mouches, 1938). Mais les années qui suivent immédiatement la Seconde Guerre Mondiale sont celles qui voient le mot, et donc la chose «science-fiction" débarquer dans la culture française ${ }^{1}$. Pour tous ceux qui le traduisent, l'éditent et le diffusent alors, c'est un genre «nouveau » et "américain »; Jean-Marc Gouanvic a très justement rappelé à quel point le choix de ne pas traduire l'expression "science-fiction ", ce qui impose en français un ordre déterminatif troublant entre les deux mots, était « le signe d'un certain rapport de la "culture française" avec la "culture" américaine.» (Gouanvic, 1999: 10) Le transfert culturel est en effet beaucoup plus qu'une simpliste «invasion » d'une culture par une autre; dans son absorption de cette dernière, la culture française adapte, modifie, s'approprie ; elle révèle ainsi certains de ses axes structurants.

5 Dans le contexte brièvement esquissé ci-dessus, on observe une mutation évidente de la situation «feuilletée » d'avant 1914 que décrit Letourneux : «trois strates fictionnelles engagées par le récit de guerre future : 1870, source référentielle fournissant la trame narrative à conjurer, l'époque de rédaction, questionnant le rapport de forces actuel des belligérants et un avenir romanesque ", où il s'agit de "clore fantasmatiquement la séquence $1871 »$ (Letourneux, 2015 : 234). La fiction de guerres futures en France après 1945 se fabrique désormais à partir de, mais aussi contre (parfois) un "genre américain " qui en structure les récits; le traumatisme d'Hiroshima est l'événement alpha autour duquel se réorganise le thème de la guerre dans la science-fiction. Mais son importation 
en France ajoute à cet événement crucial les problématiques locales de l'Europe après Yalta et, bientôt, d'une décomposition des Empires coloniaux dont la chronologie est suivie de près par le renouvellement culturel des générations du baby-boom, qui aboutit dans les années 1960 à la synthèse contestataire de 1968 et du terrorisme de gauche des " années de plomb ». La thématique insurrectionnelle s'impose ainsi dans une certaine science-fiction française, d'une manière plus politisée que dans les récits d'émeutes urbaines de la science-fiction américaine (marquée davantage par les problématiques superposant la question raciale et la question urbaine). L'opposition de cette sciencefiction contestataire à la « science-fiction militariste » structure la fin des années 1970 en France, et oppose une forme de guerilla urbaine, dont les armes sont bricolées et qui porte des accents révolutionnaires, à une guerre plus classique (armements industriels, états-majors bureaucratisés et lieux d'intrigues de pouvoir).

La social-démocratie, la fin de la Guerre Froide et l'Europe des Dix, qualifiés par certains de "grand cauchemar des années 1980 » (Lanuque), voient la guerre en science-fiction adopter de nouvelles orientations. Jean-Guillaume Lanuque en fait état ainsi :

Le regard de la science-fiction d'alors ne porte pas uniquement sur la France, mais également sur la géopolitique mondiale et les grandes puissances. (...) l'un des romans les plus caractéristiques est Le Serpent d'angoisse (1987) de Roland C. Wagner. Les États-Unis mis en scène sont comme l'exagération de ceux de Ronald Reagan. La religion protestante, l'État policier, la crise économique et l'accroissement des inégalités ont pris une telle ampleur que le pays a volé en éclat, entre des Wasps plus agressifs que jamais face aux différences (ils sont d'ailleurs surnommés les Amérikkkains), et les diverses minorités de la population, entrées en sécession. On trouve également une Commune, ici Detroit, qui s'est choisi Jack London comme symbole, profondément militarisée en raison du contexte hostile auquel elle doit faire face, déclinaison du sort de bien des révolutions du passé. Autre élément intéressant, une URSS qui s'est libéralisée, à travers laquelle on devine l'ombre portée de la perestroïka, provoquant la radicalisation en retour des États-Unis, prêt à manipuler de pseudo-terroristes d'extrême gauche pour décrédibiliser la 'patrie $\mathrm{du}$ socialisme'. On peut peut-être voir dans ce dernier point l'influence de la situation italienne des années de plomb, avec le rôle joué par la CIA. Quant à Colette Fayard, dans la nouvelle éponyme de son livre Le Jeu de l'éventail (1992), elle décrit la fin tragique des potentialités ouvertes par l'émergence de pouvoirs psychiques, avec l'utilisation de ces derniers dans un sens d'affrontement géopolitique fort classique, Mongolie et Chine alliées contre une Russie qui subit un remake du joueur de flûte de Hamelin (Lanuque, 2013 : §17).

7 Les motifs de l'insurrection se maintiennent pourtant, et s'hybrident avec ceux du terrorisme international, ce qui a pu faire dire à Goimard que «certains avancent que la troisième guerre mondiale a effectivement commencé, qu'elle est et restera une guerre en miettes; elle a sa vie quotidienne, qui fut dans les années 60 et 70 le thème central de la science-fiction contestataire » (Goimard, $1985: 12)$ : on y reviendra. La fin de l'« équilibre de la terreur » modifie évidemment cet imaginaire de la bombe qui a clivé la guerre science-fictionnelle entre un avant et un après-Hiroshima. Mais de même que le space opera n'a pas disparu avec ce traumatisme (et s'est même vu renforcé par la conquête spatiale), le post-nucléaire perdure après que le processus de limitation de la prolifération nucléaire est mis en place. Armes chimiques et bactériologiques, catastrophes écologiques investissent le thème post-apocalyptique désormais classiquement structuré en genre romanesque, avec ses invariants et ses scénarios topiques. Le thème de la guerre psychologique est plus neuf; il invite à rester attentif à l'invention science-fictionnelle que l'écrivain écossais Iain M. Banks a résumé dans son 
titre Use of Weapons (1990). En faisant notamment dériver son modèle impérial-militariste vers un modèle anarchiste-rhizomatique, Banks accomplit dans tout son cycle de la Culture $^{2}$ une spectaculaire revisitation du space opera, dont l'influence sur les romanciers français est incontestable.

\section{L'après-guerre totale : la bombe et l'espace}

En publiant dès 1946 Et la planète sauta..., B. R. Bruss prend d'emblée au sérieux le motif de la bombe atomique. Pourtant, son roman ne s'inscrit pas dans la veine naissante des fictions post-nucléaires, mais dans celle, bien plus rodée, des fictions de mondes perdus. Dans une structure classique en trois temps, il raconte comment trois savants découvrent un document dans une météorite tombée dans un champ, puis offre au lecteur le texte de ce document, en l'occurrence journal intime d'un habitant d'une planète disparue à une époque «fabuleusement ancienne » (Bruss, 1946 : 54) ; enfin, un épilogue tire les leçons de la deuxième partie. Deux extraits montreront comment ce roman a pu structurer les thèmes guerriers juste avant que la France ne réalise l'importation de la «sciencefiction » en la croisant avec le modèle de l'anticipation scientifique française d'avantguerre. Car c'est de cette dernière que ressortit le roman de Bruss; son schéma narratif pose d'emblée de jeunes hommes passionnés de science expérimentale et de découvertes, frères jumeaux des héros de Maurice Renard dans Le Péril bleu (1912). La première partie soigne tous les détails d'un récit de découverte de type archéologique, de l'extraction des coffres incrustés dans la pierre au déchiffrage des "rouleaux» et de la langue extraterrestre qu'on y trouve. La lecture est soutenue par des péripéties de type logique et scientifique, comme chez le Poe du "Scarabée d'or» où la chasse au trésor est finalement subsumée dans le défi du déchiffrage. Opportunément, les savants de Bruss découvrent le journal de celui qui a fait exploser sa planète grâce à une arme inconnue, le dragorek:

Je ne doute pas, pour ma part, qu'il s'agisse de la puissance libérée par la désintégration atomique dont nous savons nous-mêmes quels peuvent être les monstrueux effets. (...) Je présume qu'il était finalement parvenu à dissocier les atomes, non seulement de certains corps comme l'uranium ou le plutonium, mais de n'importe quel corps, et qu'il lui a suffi d'introduire l'« échelle désintégrante » dans un caillou quelconque pour faire sauter Rhama. (Bruss, $1946: 200$ )

Jamais encore le problème de l'avenir de notre espèce ne s'était posé avec autant de pressante acuité. (...) Tout près de nous entre Jupiter et Mars, errent des astéroïdes (...). Mais ce sont les débris d'un astre mort. Méditons sur le sort des Rhaméens. Craignons qu'il ne soit le nôtre. (ibid. : 203)

On est donc ici dans une veine anticipatrice / scientifique, mais orientée par un message moraliste, typique de la production du premier $\mathrm{xx}^{\mathrm{e}} \mathrm{s}$. et de ses "mondes perdus", variante populaire de la fort ancienne mélancolie des ruines. Vingt ans plus tard, René Barjavel reprend le motif de la découverte d'une civilisation très ancienne qui s'est autodétruite par la guerre dans La Nuit des temps (1968); cette fois c'est une nouvelle version de l'Atlantide qu'il situe sous l'Antarctique, et ce sont des survivants « congelés » que l'on réveille et dont on apprend l'histoire (avant qu'ils disparaissent à nouveau tragiquement). Mais il est significatif que le roman de Barjavel présente de nombreux points communs avec une fiction de monde perdu de Ridder Haggard (When the World Shook) et, surtout, un roman de Erle Cox, Out Of Silence - tous deux datés de 1919. Là encore, c'est dans l'imaginaire du premier $\mathrm{xx}^{\mathrm{e}}$ siècle que l'on puise ; et le ressort narratif 
le plus marquant de Barjavel est le tragique shakespearien qui lie les deux amants « atlantes » dans la mort, plutôt que la guerre totale dont ils sont les rescapés.

La Guerre Mondiale $n^{\circ} 3$, de Jacques Spitz, est restée inédite; le manuscrit ne doit sa publication récente (2009) qu'aux soins d'un érudit. Spitz écrit ici, probablement vers 1948 (mais certainement avant la Guerre de Corée) une sorte de pochade théâtralisant avec force humour noir un décalque de Blitzkrieg, tout juste remis au goût du jour et de la conférence de Yalta : "Le rideau de fer se lève » (Spitz, 2009: 239) cette fois-ci sur les Soviétiques qui mènent la conquête du monde, Amérique et Afrique comprises. Des armes inédites sont inventées, sans aucune plausibilité : fusées V3 en forme de palet rotatif qui pleuvent sur l'Angleterre, canon à ultrasons réduisant la population britannique à l'état végétatif, microbe sélectif donnant un hoquet mortel, trou dans la couche d'ozone, basculement de l'axe planétaire générant une glaciation ciblée de Moscou et ses environs. On le voit, c'est bien plutôt la satire qui préside à ces inventions. De même, les données géopolitiques décalquent étroitement les forces en présence vers 1945, sans en projeter aucune évolution. Avant-guerre, Spitz avant publié, avec La Guerre des mouches, un petit roman inspiré d'une nouvelle de Wells ${ }^{3}$, mais développant surtout une satire similaire du monde en 1938, où les discours même de Mussolini ou Hitler, explicitement nommés, se reconnaissaient aisément sous la caricature. Sa Guerre Mondiale $n^{\circ} 3$ est du même type, et ne trouve aucun éditeur.

Si la satire d'une guerre terrestre (trop terrestre ?) reste dans le tiroir de Spitz, les conflits spatiaux se succèdent, en revanche, au fil des rotations rapides (un ou deux romans par mois) des toutes nouvelles collections de l'époque, qui « découvrent » la science-fiction - entendons ici : la science-fiction américaine, un genre perçu et vendu comme nouveau par "Le Rayon fantastique » chez Gallimard ou «Présence du Futur » chez Denoël. Ce modèle de récit s'est structuré dans les années 1930, à partir à la fois du roman d'aventures en mer, des récits de la guerre moderne et de ses armements industriels (les fusées) et de l'épopée de l'aviation; dans le cadre des pulp magazines, E. E. Smith, par exemple, a posé avec le cycle du Fulgur les bases de ce qui devient peu à peu le space opera. Multiples races peuplant la Galaxie, expansionnisme, alliances et conflits entre elles animent ces cycles de récits. La guerre interstellaire y est un invariant. Dans la France d'après-guerre, les traductions de ces récits trouvent un public nombreux et, presque tout de suite, des imitateurs. La collection Fleuve Noir Anticipation, tout en publiant une science-fiction proclamée comme "populaire » et "française ", adopte et adapte ces invariants, sous la plume de Richard-Bessière ou B. R. Bruss; et en 1954, Francis Carsac, un Français, est publié dans "Le Rayon fantastique ", qui jusque-là ne proposait que des traductions. Si le clivage reste toutefois assez net entre les collections, les guerres contre les extra-terrestres s'y distribuent également. Simon Bréan voit plutôt dans l'uniformité stylistique et thématique la marque, au Fleuve Noir, d'une dynamique fictionnelle passablement fermée (Bréan, 2012: 107). Sans surprise, les conflits qui rythment ces récits sont pris dans un climat étouffant transposant la Guerre Froide, ou recourent aux voies de l'espionnage : L'Homme de l'espace, de Jimmy Guieu (1954), en est un bon exemple. La même année, Ceux de nulle part, de Carsac, propose un schéma que Simon Bréan analyse ainsi: "Cette grande guerre n'est pas pour autant terminée lorsque s'achève le témoignage du docteur Clair. (...) La Terre apparaît comme un espace étriqué : il faut s'expatrier pour entrer de plain-pied dans l'aventure. » (ibid.: 122). Tout se passe comme si la guerre ici composait le fond global de la civilisation; Aux armes d'Ortog, de Kurt Steiner [aka. André Ruellan] (1960), développe l'idée dans un univers où 
La Guerre bleue, qui se trouve en bonne place dans la mémoire du lecteur, se double (...) d'une motivation supérieure : ce n'était pas une simple guerre, mais une crise d'ampleur cosmique, ce qui explique l'impact prodigieux qu'elle a produit sur le monde et les humains. (ibid. : 300)

\section{Les années 1960 : peace and love, dans et au-delà des Aurès}

Plus encore que la guerre, c'est l'affrontement interracial qui caractérise les conflits de la science-fiction au point de devenir un véritable topos : la rencontre avec l'extra-terrestre est toujours l'occasion de mesurer son agressivité, directement proportionnelle à son altérité. Ecrire l'alien, c'est presque nécessairement écrire le conflit. On comprend pourquoi Ce monde est nôtre, situé par Carsac plusieurs siècles après Ceux de nulle part, a été perçu comme inspiré par la guerre d'Algérie. Publié en 1960, au climax de la si mal nommée " pacification ", il met en scène un univers multiracial, mais belliqueux, où une organisation galactique (la ligue des Terres Humaines) vient résoudre les conflits de territorialité qui éclatent sur les planètes, grâce à une règle : le droit du sol et l'antériorité d'occupation. Dans ce roman, la primitivité des peuples et des coutumes se mêle avec la modernité des scènes de vaisseaux spatiaux, et la guerre se résume à des corps à corps ou des bombardements destinés à détruire les villes, voire, le cas échéant, la planète entière. L'imaginaire de "peuples premiers", et donc "primitifs» de cet univers se lit par exemple dans cette « danse de la Guerre » :

Ce soir, quand la Lune se lèvera, aura lieu la grande danse de la Guerre. Otso y participe (...). J'aimerais que vous y preniez part, toi comme notre allié d'au-delà du ciel, et toi, femme, comme le vrai chef des Bérandiens, afin de prouver à mes hommes (...) que ton peuple a enfin compris la vérité et l'horreur de sa conduite envers nous. (Carsac, [1960] 1996 : 642)

Dans l'édition complète de l'œuvre, Georges Bordes, le fils de Carsac, se fonde d'ailleurs sur les traits de cet imaginaire, renvoyant plutôt à la «brousse d'Indochine ", pour réfuter le rapprochement avec l'Algérie. Il cite une lettre de son père :

Le squelette de ce roman était déjà debout avant que la guerre d'Algérie ne commence, et j'avais en tête la guerre du Vietnam (première phase). (...) je connais le Vietnam, le Cambodge et le Laos, pays que j'aime beaucoup, et que j'ai vus déchirés par des guerres inutiles. De même, la «brousse » dont je parle dans divers livres n'est point la brousse africaine, que j'ignore, mais celle de l'Indochine, assez différente. Je sais que les Français n'ont pas la «tête asiatique » et sont plutôt tournés vers l'Afrique, c'est sans doute ce qui explique ce point de vue (que Ce monde est nôtre a été inspiré par l'Algérie. GB.). (Bordes, 1996 : 692)

On sait que la guerre d'Indochine a moins marqué l'imaginaire culturel français que celle d'Algérie; la conscription obligatoire, la proximité du territoire, le rapatriement des Harkis, l'intrication de politique intérieure et extérieure, les attentats de l'OAS, tout a contribué à faire de la "guerre d'Algérie » une "guerre française ». Outre-Atlantique, dans ce foyer littéraire de science-fiction qui continue de rayonner vers la France, la guerre du Vietnam devient, après 1963 et les premiers envois de marines en masse, la " guerre américaine » par excellence, clivant l'ensemble de la société états-unienne en ce qui prend parfois le nom de «Second Civil War ». Joe Haldeman, Robert Heinlein, Ursula le Guin, entre autres, transcrivent ce conflit, ses paysages, ses rapports de forces et ses combats typiques dans leurs récits de science-fiction (par exemple, dans The Word for World is Forest, de Le Guin, l'attaque des autochtones cachés dans les souterrains de la 
forêt vierge est immanquablement lu en résonance avec les combats contre les sections cachées des Viet Congs). En France, rien de tel ; Carsac se défend d'avoir fait allégorie, et il faut attendre 1976 pour que Gérard Klein déclare, dans la préface d'un recueil consacré aux années 1960-1964, que

mon « vrai » roman sur la guerre d'Algérie reste Les seigneurs de la guerre qui tente de suggérer, entre autre choses, que si les guerres ont bien évidemment des origines économiques et sociales, elles deviennent, sitôt déclenchées, des structures autonomes qui se nourrissent des destructions et des souffrances qu'elles engendrent et qui ne peuvent être défaites que de l'intérieur en les conduisant à se prendre pour proies et se dévorer elles-mêmes. (Klein, 1976)

De fait, Les Seigneurs de la guerre (1971) ne raconte en rien les scènes qui hantent les témoignages d'Algérie; c'est un roman sur la guerre comme civilisation globale, dont le héros, qui ne connaît du monde que la guerre, est entraîné dans différents espaces-temps parallèles afin d'annuler les conflits en recourant à des sortes de paradoxes temporels. Le labyrinthe est tellement complexe que les paragraphes dédiés à l'écriture de la guerre en ressortent, comme autant de repères tendus au lecteur, en portant une morale subtile : les reconnaître, et s'y accrocher comme à autant d'aides à la compréhension, c'est reconduire peu ou prou le mécanisme infernal d'Aergistal, "le lieu de toutes les guerres ». Au lieu de quoi, le héros est amené, en fin de compte, à

Se tendre la main à lui-même par-dessus un gouffre de six mille ans.

(...)

C'était de l'histoire ancienne. Une histoire vieille de six mille ans, réchauffée. Dans

l'avenir où il avait vécu, la guerre entre les Puissances Solaires et les Princes d'Uria

était une affaire classée. Personne ne l'avait gagnée et les deux camps, au fond,

l'avaient perdue. (Klein, $1971: 217-219$ )

Il s'agit en quelque sorte de clore la guerre grâce au voyage dans le temps. À divers titres, c'est ce que font aussi Jean-Pierre Andrevon, avec Les Hommes-machines contre Gandahar (1969), et Roland C. Wagner avec Rêves de gloire (2011). Cinquante ans après les accords d'Evian, ce dernier propose une vaste uchronie polyphonique mettant en scène une autre histoire, utopique et pacifiste, où Alger est restée une enclave française. La sciencefiction, ici, que connaît parfaitement Roland C. Wagner et dont il maîtrise tous les codes, est exploitée par son angle uchronique: très loin du space opera, des voyages dans le temps et des combats spatiaux, cette science-fiction-là est sans doute celle qui écrit le plus classiquement les topoï de la guerre la plus traumatisante de la décolonisation française: on y retrouve les "corvées de bois» de sinistre mémoire (exécutions sommaires), les scènes de torture à la "gégène " (générateur électrique), les discours racistes des colons, les manifestations de rue dégénérant tragiquement (ou non), les massacres des villageois de l'Aurès. Le procédé uchronique prend alors valeur de « voyage dans le temps " par l'écriture, et réécriture de l'Histoire. Des extraits d'études et de discours fictifs apparaissent en exergue de chaque partie; les topoï traumatisants sont entrelacés avec l'imaginaire d'une communauté progressiste et non-violente installée dans l'enclave algéroise. Les uchronies depuis 2000 ont beaucoup de succès; les batailles, les guerres sont leurs points de divergence historique favoris, comme dans Tancrède de Bellagamba (2009), Le Déchronologue de Beauverger (2009). Cette vogue de l'uchronie estelle l'indice d'une impuissance à agir dans le monde réel, et du fantasme collectif d'une réécriture possible des événements tragiques de l'Histoire? On a pu commenter ainsi, dans l'Angleterre des années 1960, le virage de la science-fiction vers la fantasy (avec Moorcock et la revue New Worlds) et les «mondes intérieurs " (inner spaces) de Ballard: mélancolie morbide de l'Empire britannique finissant, évasion dans des hors-mondes 
merveilleux où un héroïsme topique est encore possible. Rêves de gloire, en tout cas, écrit à la fois une sorte de catalogue des souffrances algériennes, un répertoire d'images et de matrices narratives typiquement attachées à ce conflit, et traverse par l'écriture le feuilleté des événements advenus et non-advenus.

En 1969, pour y revenir, Andrevon recourt lui aussi au voyage dans le temps, mais au sein de la diégèse, de façon similaire à Klein (qui écrit en 1970) : un héros est chargé de rectifier le cours des événements. Le récit est toutefois beaucoup plus simple à suivre que dans Les Seigneurs de la guerre: Gandahar, une planète pacifique, un peu hippie, où l'on a limité l'usage des machines et où les habitants vivent de plaisirs et de volupté en se déplaçant sur des insectes géants, se voit envahir par des "Hommes-machines" destructeurs. En voyageant dans le temps, le héros, Sylvin Lanvère, parvient à comprendre qu'ils sont le fruit des mauvaises créations du Métamorphe, sorte d'intelligence artificielle ; en voyageant dans le temps, il parvient à les éradiquer. Le style des scènes de combats parvient habilement à distinguer les êtres machiniques et les êtres naturels : «Imagine une armée de fourmis, grandes comme des hommes, debout sur leurs pattes de derrière, cuirassées comme des polomoches et lançant devant elles un réseau serré de flammes.... » (Andrevon, 1969 : 148).

Sylvin Lanvère se préoccupa avant tout de manier mentalement une ligne de vol rectiligne et horizontale au frelon, dont la course était empreinte de fantaisies incontrôlées. (...) il poussa un sifflement de surprise en observant le manège des oiseaux, qui s'étaient alignés de manière presque militaire, et formait autour du chevalier et de sa monture comme un grand $\mathrm{V}$ dont la pointe était loin devant la tête du frelon (ibid. : 173)

dans une imagerie originale et très picturale ${ }^{4}$. Mais surtout, la fin du roman, tournant le dos aux facilités du "paradoxe du grand-père $"^{5}$, s'engage résolument dans une contestation de l'héroïsme guerrier :

Ainsi, il n'avait jamais été convoqué par la Reine, il n'avait jamais revu Blanminor, il n'avait jamais... Il n'avait jamais rien fait, il n'avait jamais sauvé Gandahar, il n'était pas un héros. Un vieux relent collégial le lui fit regretter un instant. Mais guère plus d'une seconde : «Heureux sont les peuples qui n'ont pas besoin de héros. » (ibid. : 208)

18 Une morale s'en dégage, parfaitement en accord avec les mouvements anti-militaristes des années 1968 et suivantes; la clausule des Seigneurs de la guerre est moins clairement exportable à un autre imaginaire que celui de la science-fiction et des univers parallèles, mais exprime une idée similaire :

Quelque part sur Uria, dans six mille ans, un autre Corson essayait de survivre. Il ignorait encore qu'il effacerait un conflit sous le regard froid des ères (...)

- Pourquoi moi ? se dit Corson en reprenant le chemin de l'avenir. (...)

Mais il demeura sur le seuil, hésitant, trébuchant, car le temps n'avait pas encore fait son oeuvre, ni l'expérience, et parce que ces Corsons-là commençaient tout juste d'avoir l'ombre d'une chance (Klein, 1971 : 223)

où un lecteur de 1970 ne peut pas ne pas entendre les mots de John Lennon et Yoko Ono, devenus le slogan des manifestations anti-guerre du Vietnam aux États-Unis: «Give Peace a Chance ».

C'est également sur un message pacifiste que se termine l'un des romans les plus importants de la science-fiction française, Oms en série de Stefan Wul (1972), popularisé par le film d'animation de Topor et Laloux, La Planète sauvage (1973). On y suit le parcours des «oms », lointains descendants des Humains devenus animaux de compagnie d'une race extra-terrestre, lorsqu'ils se révoltent contre leurs maitres Draags, entrent en 
guerre, puis signent un traité de paix avec eux. Un équilibre subtil est tenu ici entre le message pacifiste et la conscience de la violence : Oms en série n'est pas un roman niais sur une guerre mièvre, et offre quelques scènes cruelles; mais le principe d'une guerre défensive est attribué aux héros, par exemple, ce qui contraste vivement avec le style de leurs ennemis Draags (pourtant loin d'être barbares) : «- Je vous propose d'envoyer au port une escouade de militaires cuirassés. Ils auront l'ordre de tout balayer aux rayons durs si les oms leur font mauvais accueil. Nous pourrons ainsi visiter la cité morte (...)» (Wul, 1972:124)

Uni à Andrevon par son pacifisme, Wul contraste toutefois avec lui par la dynamique ouverte de son excipit: là où Sylvin Lanvère se contente de déclamer une formule terminale, Terr, le petit «om » bien nommé, conclut le roman sur la signature d'un traité organisant le principe d'une « saine émulation » entre les deux races :

- Draags, dit-il, et vous, petits oms, j'ai signé ! Le travail de vos Ediles est terminé. la mise au point des détails sera votée par les conseils. Nos deux races sont unies pour le meilleur et pour le pire!

Dans le soir doré descendant sur la mer, deux vaisseaux s'accotaient l'un à l'autre, comme deux amis. Des hymnes draags et des chants d'oms ondulaient dans la brise. (Wul, 1972: 186)

21 Proche écho de Ce monde est nôtre, qui voyait les races de la planète Nécat se rapprocher, après des siècles de haine mutuelle, Oms en série propose le schéma narratif d'un récit démocratiquement ouvert sur son passé (ce qui a conduit les Oms à régresser) et, programmatiquement, sur son futur (ce qui peut aider les Draags à ne pas régresser à leur tour). Le pacifisme réel se lit dans cette confiance en l'amitié interraciale, et ses développements pleins de surprise, plus encore que dans une promesse d'absence de guerre (jamais exprimée). En ce sens, ce sont des anti-Boulle: dans La Planète des Singes (1963), la méfiance des races humaine et simiesque est le coeur immuable de l'hostilité belliqueuse, quelles que soient les bonnes volontés de quelques hommes ou singes. Le roman de Pierre Boulle, dans son texte-même, est construit sur un emboîtement de récits enchâssés et sur des effets de surprise qui signent une poétique du choc, de la limite et de l'éternelle lutte de l'un contre l'autre. Devenue en près de cinquante ans une des franchises médiatiques hollywoodiennes les plus rentables, Planet of the Apes décline depuis 1968 (date de la première adaptation du roman) cette impossibilité structurelle de mêler les races, où la guerre entre hommes et singes est l'horizon d'attente indépassable d'une recherche $d u$ « propre » de chacun.

\section{Après 1968 : l'insurrection et son échec}

Les années 1970 voient clairement la science-fiction française opérer sa grande diversification. En regard des précédentes écritures de la guerre, il suffit d'un exemple puisé au Fleuve Noir Anticipation pour mesurer l'écart entre ces science-fiction pacifistes et une science-fiction qui l'est moins, et par-là même, peut-être, désigner ce que l'on nomme désormais la «science-fiction militariste ». Voici l'une des premières pages d' Azraëc de Virgo :

Seul, toujours seul, inapte à perpétuer ma race. Cloîtré à jamais. O liberté ! A quoi bon vivre? (...) Moi, le dernier de ma race, le parfait, le tout-puissant, cette prison me rendra fou! (...) Je reproduis à ma guise la fraîcheur du matin, la moiteur de l'orage, les embruns des vagues, mais il ne s'agit que de fallacieuses apparences! J'ignore tout du monde réel. Pourquoi suis-je haï de tous? Pouvoir, puissance, 
domination, à quoi bon? Je souffre, mon coeur se brise. Ah! si je pouvais briser cette carapace, sortir mon corps difforme de son carcan. L'azur du ciel me manque tant. Oh! ma tête.

(...) Azraëc le puissant, l'unique, cyborg souverain des 3000 galaxies florissantes de l'amas de Virgo, était en pleine crise de dépression. Pourtant, l'homoncule se relaxait lentement, ses membres ténus cessaient de se tordre en tous sens. (...)

Azraëc, l'homoncule aux membres chétifs, au cerveau hypertrophié, Azraëc le génie, le chef des légions de robots, et du Grand Corps des astrots galactiques, insatiable d'ambition, subtil et tortueux, faux et machiavélique, Azraëc s'ennuyait dans sa prison de titane.

Un seul remède : la guerre. Jouir des souffrances des autres, conquérir, dominer.

(Barbet, $1971: 7-8$ )

Dans cette écriture étrangement riche de motifs (le cyborg dépressif, qu'un Philip K. Dick eût traité méditativement), mais qui les délaisse au profit d'une guerre perçue, en tout sadisme assumé, comme un simple divertissement - et comme seul ressort narratif - on repère à coup sûr un type de science-fiction qui sera bientôt la cible d'une contestation violente issue du milieu science-fiction lui-même (et principalement d'Andrevon). On repère peut-être aussi une prise de conscience plus profonde, que certains opus du «nouveau space opera », vingt ans plus tard, formuleront plus explicitement à l'orée de l'an 2000. On repère quoi qu'il en soit, et à coup sûr, la raison de la mauvaise réputation de la science-fiction auprès de ceux qui la connaissent mal : à l'aune d'Azraëc de Virgo (et des volumes à rotation rapide qui lui ressemblent, et qui sont les plus nombreux à tomber sous l'œil du chaland dans les librairies, gares, boutiques diverses), la science-fiction est assurément un genre assez pauvre qui se confond avec des récits conventionnels de guerres dans l'espace.

Vers 1975, dans le sillage de toute une contre-culture alimentée par les mouvements hippies, écologistes, antimilitaristes, tiers-mondistes, communautaristes, la sciencefiction française connaît une floraison contre-culturelle qui s'exprime notamment en bande dessinée (dans les revues Actuel, puis dans Métal Hurlant). En littérature, des auteurs se regroupent autour de Jean-Pierre Andrevon dans trois recueils intitulés Retour à la Terre (1975-1977). L'un de ceux qui participent au troisième recueil n'est autre que Bernard Blanc, dont le livre Pourquoi j'ai tué Jules Verne, l'année suivante (1978), se présente ainsi : «Fini, les histoires de fusées. Fini, les conquêtes spatiales et la guerre des étoiles. Les écrivains de science-fiction d'aujourd'hui parlent de leur présent et de leurs angoisses, après avoir effectué un symbolique, mais très réel, retour à la Terre. » (Blanc, 1978: $4^{\mathrm{e}}$ de couverture). C'est également Blanc qui fonde la revue Alerte! en 1977 et patronne, chez le même éditeur Kesselring, d'autres collectifs d'auteurs militants. Leur premier éditorial vaut d'être cité parce qu'il évoque bien comment l'idéologie, les techniques narratives, et le motif guerrier sont intriqués dans la science-fiction:

Français jusqu'aux bouts des ongles. La SF d'ici ne sera pas une succursale de l'OTAN. (...) Autour de vous, le ciel est lourd et le béton froid. Faut-il vous mentir et vous raconter des histoires d'amour sur fond de musique douce, en technicolor publicitaire? Faut-il vous dire que la race humaine est splendide et ses dirigeants magnifiques? Faut-il vous montrer des fables où les extra-terrestres sont laids et dangereux? La SF a longtemps joué ce rôle d'usine à rêves, avec ses fantasmes réactionnaires et ses putasseries d'amuseuse publique. Une poignée d'écrivains français a décidé de renverser la vapeur (...). S’ils faisaient dans le grand spectacle sanguinolent du génocide extra-terrestre, dans le film catastrophe plein de sauveteurs musclés (...) alors là, ils se rempliraient les poches. (...) Aujourd'hui, la SF casse les mythes et incendie les fusées. Aujourd'hui, elle parle des flics et de l'armée et explique qu'ils sont du mauvais côté, du côté des exploiteurs et des tyrans. Elle 
parle de la pollution et nomme les responsables : Rhône-Poulenc, EDF, et Péchiney.

ATTACHEZ VOS CEINTURES ! LA SF VA ATTERRIR ET ÇA VA SAIGNER ! (Ciel lourd...,

$1977: 10-13)$

On l'aura bien compris, cette « Nouvelle Science-Fiction Française Politique » (NSFFP) est tout sauf non-violente. Son écriture de la guerre, toutefois, recourt clairement aux topoï de la guerre civile, de la guerilla, et de l'insurrection. Les personnages sont installés dans la clandestinité, les marges urbaines et sociales, les communautés militantes; la pratique du secret emprunte à l'espionnage, mais le militantisme requiert la diffusion des thèses et des discours ; cela conduit à un étonnant mélange de procédés, favorisé par l'explosion des médias à cette époque : certes, on trouvera force dialogues philosophiques retraçant des débats de comité ou des initiations politiques (régulièrement associées à des initiations sexuelles, comme un peu partout à l'époque de la libération sexuelle); mais aussi des procédés de collage et de montage de textes journalistiques, d'émissions de radio, de publicités, tracts et slogans, rompant ou au contraire soutenant la narration. Tous à Zanzibar, de John Brunner (1968), traduit en français en 1972, est le modèle de ces pratiques. De la sorte, la langue et le style, les canaux de communication sont aussi bricolés que les armes ou les groupes de combattants. C'est à peu près ce que défend Pourquoij'ai tué Jules Verne : pour en finir avec la science-fiction capitaliste et militariste, il faut aussi écrire autrement. «Casser du flic » et casser la langue de la science-fiction vont de pair.

On peut comprendre alors en quoi cette science-fiction politique, dont le relatif échec est diagnostiqué par Andrevon lui-même, en 1979, a pu être considérée comme une avantgarde, et rapprochée, du coup, de l'avant-garde formaliste du groupe «Limite » au milieu des années 1980 ; en 2004, un débat confronta Roland C. Wagner à Christophe Ruffin pour savoir si la NSFFP avait détournée de la science-fiction française son lectorat ${ }^{6}$. Il n'est pas nécessaire de le trancher pour observer en quoi un auteur comme Antoine Volodine, qui commence sa carrière dans les années 1980 chez "Présence du Futur », participe du groupe «Limite» en exploitant les thèmes insurrectionnels. Son écriture dépasse largement la science-fiction, dont il quitte d'ailleurs la communauté éditoriale en 1990 ; elle construit petit à petit ce qui deviendra le "post-exotisme" à travers des visions futuristes de combattants révolutionnaires emprisonnés après l'échec de l'insurrection. Dans cette écriture de la guerre, ce ne sont pas les scènes de combat qui sont structurantes, mais les scènes de torture, de misère carcérale, de terreur urbaine et de répression policière. On est bien dans un " après-guerre ", mais où la reconstruction reste hantée par les méthodes d'une guerre «sale » puisant ses images dans le terrorisme des "années de plomb», la police politique des dictatures et la phraséologie des groupes armés des années 1970 :

Citoyennes, camarades, rendre hommage à Slobodan-Kateth Minahualpa revient à rendre hommage à sa longue vie de combattant révolutionnaire au service de l'esprit et de la culture. (...) je vous invite à méditer sur les réponses si humaines qu'il apportait à la si inhumaine énigme d'une présence non terrestre parmi nous (Volodine, 1985 : 73)

L'effet critique porté sur les fictions de guerres spatiales n'est pas mince : leur apparence héroïque, leur « propreté » assistée par ordinateur en seront durablement décrédibilisées.

Le « post-exotisme » de Volodine, qui inspire aujourd'hui des auteurs comme Leo Henry et Jacques Muschielli (Yama Loka Terminus en 2008, Bara Yogoï en 2010), s'est aussi conjugué à une vague de fictions mettant en scène une France post-nucléaire, notamment dans le $9^{e}$ art (Marseil, de Crespin, 1979); le recueil Avenirs à la dérive, qui devait justement 
être le «Retour à la Terre $n^{\circ} 4$ » et se voit édité chez Kesselring après le refus de «Présence du Futur », adopte ce thème pour clore l'aventure de la NSFFP :

Décrire le chaos? Réunir douze auteurs pour le pire (plus que pour ce "meilleur » inaccessible)? Jeter aux orties toutes nos illusions pour essayer de conserver «l'espoir"

C'est toute la démarche de cette anthologie.

Nous avons tous, dans un coin de notre cerveau, les images de notre apocalypse. Tous.

Alors ne plus partir pour une galaxie lointaine, mais connaitre notre terre pour la mieux vivre; retrouver cette planète en la sachant fragile, en danger de mort.

Rester pour vivre. (Andrevon, 1979 : préface)

La fin des années 1970 voit ainsi se rejoindre, dans une appropriation culturelle française, les deux courants d'une écriture de la guerre en science-fiction que nous identifiions plus haut: les combats spatiaux, le post-nucléaire. Le second motif, en France, a davantage prospéré sur la contestation du nucléaire civil que sur l'angoisse de la $3^{e}$ guerre mondiale ou la sidération d'Hiroshima. Il s'épanouit dans cette science-fiction écologiste, contestataire, qui mêle la critique du capitalisme et la défense des minorités, le retour à la ruralité contre la destruction urbaine du lien social. Mais dès lors que le post-nucléaire (civil ou militaire) devient un genre en soi, il codifie la fiction avec ses motifs propres et ses lieux attendus: routes désertées gagnées par la végétation, villes abandonnées, groupes humains de petite taille et d'organisation tribale. Des idéologies diverses l'investissent; l'exaltation de la violence comme seule loi restant active après la catastrophe s'épanouit au "Fleuve Noir Anticipation", par exemple dans L'Autoroute sauvage de Gilles Thomas (1976) ou L'Ombre dans la vallée et Le Viaduc perdu de Jean-Louis Le May, l'année même où le cycle de « Mad Max » démarre au cinéma (1979).

\section{Après 1981 : « grand cauchemar » social-démocrate et nouveau space opera}

La science-fiction française entre, avec la victoire du Parti Socialiste à l'élection présidentielle de 1981, dans ce que certains ont appelé le "grand cauchemar des années 80 » (Lanuque, 2013). L'écriture de l'insurrection révolutionnaire, désactivée par l'arrivée au pouvoir de la gauche, y subit, selon cette analyse, un coup fatal, qui expliquerait peut-être aussi la conjugaison de ses motifs littéraires avec ceux de la fiction post-nucléaire. De même, la construction européenne, qui généralise la social-démocratie réformiste, est diversement interprétée par la science-fiction ; pour Philippe Curval, elle appelle tôt ou tard une dystopie feutrée qu'il met en scène dès Cette chère humanité en 1976, et dont confirme l'entropie en 2009 dans Lothar Blues. Mais pour Georges Panchard, l'Europe et son idéologie sont carrément responsables d'une atroce guerre civile entre Européens et Musulmans dont la narration, à coups de récits de viols collectifs par les Barbus et d'atrocités urbaines, est l'un des fils rouges du roman Forteresse (2005) :

Il semble difficile de comprendre comment un courant de pensée aussi furieusement imbécile et suicidaire que la Correction politique a pu se développer. (...) La meilleure définition que l'on puisse donner de la Correction politique, c'est qu'elle fut le fascisme des bons sentiments. La Correction fut la concrétisation d'un puritanisme de gauche, prétendument centré sur des valeurs qu'il affirmait défendre alors qu'il ne cessait de les dévoyer, confondant allègrement éthique et censure, humanisme et candeur, progrès et régression.

En culpabilisant jusqu'à l'idée de résistance, en sapant le potentiel naturel de 
défense de la civilisation occidentale, la Correction a créé les conditions de la guerre (...) (Panchard, 2005 : 28)

31 stées, et qui peuvent valoir comme transition vers la période de "l'extrême contemporain ». Pierre Pelot, d'une part, publie La Guerre olympique, dystopie organisant désormais les conflits entre Nations à travers des jeux meurtriers dont les gains et défaites se traduisent par des exécutions en masse dans les camps de prisonniers. Parfaitement peu plausible, cette allégorie ultraviolente du sport et de la guerre se construit sur un schéma géopolitique encore tendu entre blocs étatiques cohérents, et exploite le motif d'une classe dirigeante surpuissante assistée par des ordinateurs qui leur permettent de contrôler les peuples à travers les médias. Jugé simpliste à sa sortie, ce roman, qui prend le relais d'une longue tradition américaine, a anticipé avec justesse, non pas l'état du monde actuel (heureusement !), mais le succès médiatique des cycles comme Hunger Games (depuis 2008), qui y puise son inspiration.

D'autre part, Jean Hougron, connu pour son cycle de La Nuit indochinoise, mais dont le seul roman de science-fiction, Le Signe du Chien (1961) ne traitait pas cette matière, publie un space opera, Le Naguen, chez Plon, hors du circuit science-fiction. Affichant une parfaite maîtrise des codes du roman de guerre et d'espionnage, il suggère une lecture à clés, évoquant peut-être l'invasion de l'Afghanistan; mais il exploite surtout, narrativement, une amnésie du héros, et une répartition sans manichéisme des idéologies entre les races combattantes. Vaste fresque de politique avant tout, ce roman de guerre stellaire marque un jalon vers le nouveau space opera, où l'on verra, dans les années 1990, se disloquer les idéologies trop simples et se multiplier les questionnements sur la guerre. L'Ecossais Iain M. Banks, avec par exemple Use of Weapons (1990), met également en scène un amnésique, dans un roman où les chapitres de combats sont comme les étapes d'une psychanalyse. J'ai montré ailleurs ${ }^{7}$ en quoi cette formulation pouvait se lire comme une écriture de la mauvaise conscience du space opera après le simplisme militariste; deux branches de la science-fiction française illustrent comment Le Naguen peut faire transition.

Dans l'une, l'écriture de la guerre se coule dans une spiritualisation marquée et un héroïsme d'équipe qui portent son développement superlatif. C'est le cas dans Les Guerriers du silence de Bordage (1993), qui narre en 1636 pages la longue marche et convergence de 12 "guerriers inddiques " destinés à combattre "l'Incréé " dans un brillant récit de space fantasy sans rapport avec notre univers, mais constamment émaillé de références religieuses. Une spiritualisation comparable du lien soudant les soldats, mais totalement athée cette fois, est à l'oeuvre dans le cycle de F.A.U.S.T. de Serge Lehman (1996-1997), qui raconte comment se déroulent les conflits sur une Terre désormais gouvernée, bien avant Panchard, autant par les Puissances (industrielles) que par les États (réduits à rien) ou les Fédérations (notamment celle d'Europe). Par contre, chez Maurice Dantec, c'est l'individualisme du guerrier solitaire qui est exalté dans Babylon Babies 
(1999). Ayant élevé la guerre au rang d'un art, présenté comme poète et assassin dans une figure romantique, puissante et morbide, le héros Toorop se voit consacrer toute une première partie intitulée "Celui qui cherche et qui détruit " ${ }^{8}$, qui est comme un long poème de guerre en Europe et Asie Centrale en ouverture d'un roman qui ne verra ensuite s'affronter que des bikers montréalais. En totale opposition avec cette idéologie individualiste et cynique, un roman comme La Zone du dehors, d'Alain Damasio, inscrit en 1999 un roman utopique et insurrectionnel comme la NSFFP en aurait rêvé. On y découvre, entre mille discours deleuziens et flashs mémoriels complexifiant le fil narratif, une sorte de révolution orbitale et l'installation d'une communauté « en-dehors » de la zone protégée. Signalons toutefois que ce roman, passé tout à fait inaperçu, n'a reçu un véritable public qu'après l'extraordinaire succès de La Horde du Contrevent (2004) dont quelques chapitres formulent avec fascination la force brute et la violence extrême (des hommes ou de la nature), et ne dépareraient pas chez Dantec.

Dans la science-fiction française toutefois, une écriture comme celle de Dantec, tendue vers l'objectif de révéler le sens du monde et de la vie à travers la violence de la guerre, est concurrencée par des œuvres comme Étoiles mortes de Dunyach (1991) ou Aucune étoile aussi lointaine de Lehman (1998), qui mettent en place une

logique de disqualification de la violence [qui] informe les récits d'aventures spatiales (...) Même s'il s'agit de représenter des aventures exotiques et même des guerres interstellaires, le message général des romans de science-fiction de cette époque est que la violence n'est pas une solution. Les voies de la guerre et de la destruction se révèlent moins efficaces que la recherche de l'équilibre et de l'harmonie, ainsi qu'il apparaît dans la trilogie des Guerriers du silence (...) Les visées militaires sont systématiquement disqualifiées [dans] les AnimauxVilles de Jean-Claude Dunyach (...) Lehman revisite toutes les thématiques guerrières du space opera au cours de l'initiation d'un jeune homme se rêvant en héros de l'espace. L'artefact immensément destructeur qu'il poursuit se révèle doté de conscience (...) et finit par renoncer à la violence, si bien que l'aventure du héros se révèle être une quête de sens et non une opération militaire. (Bréan, 2012 : 257-258)

\section{BIBLIOGRAPHIE}

ALTAIRAC, Joseph (2009). « Postface », in Spitz Jacques (2009). Joyeuses apocalypses. Bragelonne. pp. 417-430.

ANDREVON, Jean-Pierre, dir. (1979). Avenirs en dérive. Berne : Kesselring. coll. Ici et maintenant Collectif $n^{\circ} 5$.

ANDREVon, Jean-Pierre (1969). Gandahar. Paris : Denoël. coll. Présence du futur. coll. Folio SF n 13. BARBET, Pierre (1971). Azraëc de Virgo. Paris : Fleuve Noir. coll. « Anticipation » n 471.

BLANC, Bernard (1978). Pourquoi j'ai tué Jules Verne. Paris : Stock.

BORDES, Georges (1996). « À propos de l'origine de Ce monde est nôtre... », in Carsac Francis (1996).

Oeuvres complètes 1. Bruxelles : Lefrancq. pp. 687-697. 
BRÉAN, Simon (2012). La Science-fiction en France. Théorie et histoire d'une littérature. Paris : Presses de l'université Paris-Sorbonne, coll. Lettres françaises.

BRUSS, René Bonnefoy (1971). Et la planète sauta.... Paris : Robert Laffont.

CARSAC, Francis (1996). Oeuvres complètes 1. Bruxelles : Lefrancq.

Ciel lourd béton froid (1977). Berne : Kesselring. Collectif $\mathrm{n}^{\circ} 1$.

GOIMARD, Jacques (1985). « Préface », in Histoires de guerres futures. Paris : Livre de poche.

GOUANVIC, Jean-Marc (1999). Sociologie de la traduction. La science-fiction américaine dans l'espace culturel français des années 1950. Arras : Artois Presses Université.

GUIOT, Denis (1987). « Guerre », in Le Monde de la Science-fiction. M.A. éditions. En ligne, URL : http://www.noosfere.org/icarus/articles/article.asp?numarticle=762 (consulté le 30 mai 2015)

HUZ, Aurélie (2015). “ À travers les supports, par-delà les publics : les voyages de Gandahar dans la science-fiction médiatique française ", in Letourneux, Matthieu, ed. La littérature de jeunesse dans le jeu des cultures matérielles et médiatiques : circulation, adaptations, mutations, à paraître.

KLEIN, Gérard, dir. (1976). «Préface », in En un autre pays. Paris : Seghers.

KLEIN, Gérard (1971). Les Seigneurs de la guerre. Paris : Laffont. coll. Livre de poche $\mathrm{n}^{\circ} 7141$

LANGLET, Irène (2010). “ Les Mythologies au miroir du temps futur ", in Recherches \& Travaux. $\mathrm{N}^{\circ} 77$. pp. 105-117.

LANGLET, Irène (2006). La science fiction. Lecture et poétique d'un genre littéraire. Paris : Armand Colin. LANUQUE, Jean-Guillaume (2013). « La science-fiction française face au "grand cauchemar des années 1980" : une lecture politique, 1981-1993 ", ReS Futurae [En ligne], 3 | 2013, mis en ligne le 01 décembre 2013, consulté le 30 mai 2015.

URL : http://resf.revues.org/430

LETOURNEUX, Matthieu, ed. (2015). La littérature de jeunesse dans le jeu des cultures matérielles et médiatiques : circulation, adaptations, mutations, à paraître

PANCHARD, Georges (2005). Forteresse. Paris : Robert Laffont.

Spitz Jacques (2009). La Guerre mondiale $n^{\circ} 3$, in Joyeuses apocalypses. Bragelonne. pp. 237-343. volodine, Antoine (1985). Biographie comparée de Jorian Murgrave. Paris : Denoël. coll « Présence du Futur».

wUL, Stefan (1972). Oms en série. Paris : Denoël. coll « Présence du Futur ». coll. Folio SF n 11.

\section{NOTES}

1. Nous adoptons ici la conviction que le nom de genre, loin d'être une simple étiquette de classement, structure un contexte de production et de réception de la fiction. Voir Macé, 2005.

2. Le cycle de la Culture regroupe plusieurs romans parus entre 1987 et 2009.

3. Wells, « The Empire of the Ants », in Strand Magazine, 1905 ; cité par Altairac, 2009.

4. Voir à ce sujet Huz, 2015.

5. Du roman célèbre de Barjavel, Le Voyageur imprudent, où le héros tue son grand-père et donc se trouve bloqué dans le paradoxe de n'avoir pas existé, donc de n'avoir pas tué son grand-père, donc d'exister, etc. 
6. Sur ce débat, voir Langlet, 2010.

7. Voir Langlet, $2006: 213-226$.

8. Allusion transparente à la stratégie militaire généralisée dans les guerres contemporaines après la guerre du Vietnam : « Search and Destroy ».

\section{RÉSUMÉS}

À partir de la définition de la science-fiction comme genre privilégiant la guerre dans deux sousgenres majeurs, on observe comment le corpus français s'est approprié cette définition américaine entre 1945 et 2010, en l'adaptant à ses problématiques propres : après-guerre et Guerre Froide, guerre d'Algérie, contestation de mai 1968, construction européenne.

From the definition of SF as a genre centered on the war through two major subgenres, we can see how the French corpus has reapproppriated and adapted this American definition into its own issues between 1945 and 2010: post-war period and Cold War, the Algerian war, May 68 events, the European project.

\section{INDEX}

Keywords : science fiction, war, France, USA, 20th Century

Mots-clés : science-fiction, guerre, France, États-Unis, XXe siècle

\section{AUTEUR}

\section{IRÈNE LANGLET}

Université de Limoges

irene.langlet@unilim.fr 